\title{
An Innovative Multidisciplinary Healthcare Model in Student Mental Health: Experience in Hong Kong
}

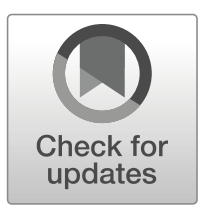

\author{
Sze Mon Lo ${ }^{1}$ (D) Ho Cheong Wong ${ }^{1} \cdot$ Ching Yee Lam ${ }^{1} \cdot$ Daniel T. L. Shek ${ }^{2}$
}

Received: 25 July 2019 / Accepted: 19 August 2019/ Published online: 24 August 2019

(C) The Author(s) 2019

\begin{abstract}
Although studies showed that common mental disorders are prevalent amongst in the university students, non-stigmatizing mental health service tailor-made to university students is not often available within the health service setting in different parts of the world. In this paper, we review the current barriers for college students seeking help from student mental health service and discuss the pros and cons of different service models. It is argued that the traditional mode of addressing student mental health issues is grossly inadequate. Based on the review, the paper also shares the pilot experiences of the Mental Wellness Clinic operated under the University Health Service of one university in Hong Kong. We also explored the strengths and challenges of this multidisciplinary coordinated healthcare model in the promotion of well-being of university students.
\end{abstract}

Keywords Student mental health · Wellness · University students $\cdot$ Hong Kong $\cdot$ Chinese

\section{Mental Health Challenge among University Students in the Global Context}

Many studies have highlighted the fact that the occurrence of common mental disorders in the community is a growing issue in the global context. According to the World Health Organization (2017), 4.4\% and 3.6\% of the global population suffer from depressive disorders and anxiety disorders, respectively. With specific reference to the Hong Kong Mental Morbidity Survey 2010-2013, Lam et al. (2015) reported that $13.3 \%$ of adults had common mental disorders. Actually, mental disorders constitute

Sze Mon Lo

dana.lo@polyu.edu.hk

1 University Health Service, The Hong Kong Polytechnic University, Hong Kong, Hong Kong

2 Department of Applied Social Sciences, The Hong Kong Polytechnic University, Hong Kong, Hong Kong 
the largest share of disability in populations worldwide (World Health Organization 2013) because young people with mental disorders are likely to continue suffering from the problems in adulthood. As three fourths of all lifetime mental cases start by age 24 years, primary prevention and secondary prevention effort need to focus on young people (Kessler et al. 2005).

In a systematic review on mental disorders in young people, Ibrahim et al. (2012) showed that $30.6 \%$ of university students suffered from depressive disorders and the prevalence rate was considerably higher than those based on the general populations. With reference to studies conducted in 21 countries differing in income levels, the World Health Organization World Mental Health Surveys estimated that around onefifth of college students showed DSM-IV/CIDI disorders in the past year. Unfortunately, Auerbach et al. (2016) showed that only $16.4 \%$ of students with disorders in the past year received any professional help in the past 12 months. Among those who received counselling, their self-reported lifetime prevalence rates of "threat-to-self" problems, such as non-suicidal self-injury, serious suicidal ideation, and suicide attempts continued to rise in the past eight years (Penn State University 2018). Suicide continues to be the second leading cause of death among college students (Turner et al. 2013). Hence, there is no doubt that university students belong to one of the high-risk groups of mental disorders, with high prevalence but low treatment rate.

With particular reference to Hong Kong, according to a web-based survey of mental distress in 7915 first-year tertiary education students in Hong Kong in 2006, Wong et al. (2006) showed that significant proportions of the students showed depression $(21 \%)$, anxiety $(41 \%)$ and moderate stress $(27 \%)$. After a lapse of ten years, Lun et al. (2018) conducted a cross-sectional study based on the responses of 1119 undergraduate students from eight universities in Hong Kong in 2016. Results showed that a high proportion of the students displayed depressive symptoms (68.5\%), anxiety symptoms $(54.4 \%)$, moderately severe to severe depressive symptoms $(9 \%)$ and severe anxiety symptoms $(6 \%)$. In fact, the worrying mental health of university students can be regarded as a manifestation of the deteriorating trend in life satisfaction and hopelessness in the high school and university years (Shek and Liang 2018; Shek et al. 2017). At the same time, several risk factors are adversely affecting the healthy development of adolescents and young people in Hong Kong (Shek and Siu 2019a, b).

Obviously, mental health issues adversely affect the well-being and positive development of university students. Mental disorders were found to be significantly associated with termination of schooling (Breslau et al. 2008), marital relationship (Kessler et al. 1998), and mortality (Osby et al. 2001). While health service is commonly established in many university campuses over the world, specialized and tailor-made mental health service (particularly non-stigmatizing services) is not often found within the health service setting. Although primary care clinicians or specialists in some University Health Services over the world do provide mental health services to students [e.g., (University of Michigan 2019), (University of Bristol 2019), (Monash University 2019), (National University of Singapore 2019), (The University of Tokyo 2019)], the mode of service operation, referral logistics, service utilization and outcome measurement are not widely discussed or published from literature search. Besides, relevant service information from China, Taiwan, and Hong Kong are limited.

Despite the economic growth and medical advances in Hong Kong over the past few decades, Shek and Siu (2019a, b) pointed out that policies, services, and research 
surrounding adolescent mental health face seven unresolved problems waiting for solutions. One of the problems suggested by the authors is that the related policies, services, and research are grossly fragmented. Against this background, this paper discusses an integrated and multidisciplinary student mental health services model operated in a local university community setting.

\section{Barriers to Mental Health Service for University Students}

Given the high prevalence but low treatment rate (Auerbach et al. 2016), barriers to mental health service utilization by university students have to be explored in order to design for an effective management proposal, which can remove the barriers. There are three categories of barriers to mental health services (Thompson et al. 2004; Wong et al. 2019):

\section{a. Knowledge-Based Barriers}

In view of the low knowledge level to mental illnesses, people have poor insight and are unable to identify symptoms (Shek 1990). Hence, they do not know that they need to seek from mental health professionals and/or they are not aware of how to get mental health services.

\section{b. Structural Barriers}

Even if people are aware of the symptoms and willing to improve, there are practical difficulties for them to seek medical help due to financial concern or inconvenience of travel to the health service centre, etc. Besides, the referral systems within the health system (such as referring a case to the psychiatrist by a social worker) may also be annoying and frustrating for the client.

\section{c. Attitudinal Barriers}

Even if people are aware of the symptoms and practically can seek for medical help, they are unwilling to do so due to the stigmas, fears and/or embarrassment related to the identity of being a mental patient. People, especially in the Chinese community, generally have low acceptance of mental illnesses (Ho et al. 2003).

\section{Different Management Proposals in the Hong Kong Context}

There are different health care systems in different parts of the world. Understanding the local context is critical when different management proposals for mental health service of university students in Hong Kong has to be discussed. It is expected that the experience in Hong Kong may serve as a useful reference for colleagues in other parts of the world.

In general, the public and private sectors are responsible for the provision of mental health services in Hong Kong (HKSAR 2019). Regarding the public sector, the Food and Health Bureau (FHB) develop health-related policies and allocate resources for 
Hong Kong's health services. Operationally, the Department of Health (DH) takes care of the public health services in Hong Kong. Unfortunately, the Student Health Service under DH only provides health programmes for primary and secondary school (Department of Health, HKSAR 2019) and the health of university students is beyond its scope of this service. Hospital Authority (HA) under the public sector is mainly responsible for management of public hospitals. HA is a major provider for psychiatric service, including inpatient services and outpatient service provided by the Specialist Outpatient Clinics (SOPCs).

The demand for specialist psychiatric service is rising in Hong Kong probably because of the growing awareness of the community about mental illnesses and the inability of primary care service to cope with the related demand. As SOPCs provide services for people with common or severe mental illnesses, its role as the main entry point into the public health care system is a stressful one because the related professionals are expected to carry out acute management as well as treatment tasks. According to Hospital Authority (2011), a key long-term strategic objective for adult mental health service is to manage common mental disorders in primary care settings. To accomplish this objective, HA has developed a shared care model where psychiatrists work together with primary care doctors to help people suffering from common mental disorders. For "Integrated Mental Health Programme for Common Mental Disorder Patients in General Outpatient Clinics, IMHP" starting since 2010 (Hospital Authority 2019a), it is run by family physicians and case workers who may be nurses, social workers or occupational therapists, with support from hospital psychiatrists. The programme recruits patients from general outpatient clinics with a significant proportion being middle to old aged groups having chronic illnesses such as diabetes and hypertension. To the best understanding, the programme does not focus on young age group such as university students with relatively good physical health.

Regarding the private sector, patients with mental problems mainly seek help from psychiatrists, family physicians, clinical psychologists or counsellors in private practice at variable consultation fees.

\section{Alternatives for Mental Health Service to University Students}

With a background knowledge of the Hong Kong health care system, the following is the discussion of the pros and cons of four proposals regarding mental health service to university students who have mental health needs.

\section{Proposal A: All Refer out to Public Psychiatrists}

If all students presented with mental health disturbances are referred to public psychiatrists for medical management, apparently the cost and responsibility of providing medical service to students will be shifted to the Hospital Authority from the university. However, psychiatric SOPCs under HA is a very busy clinic with 46,680 new cases in the year of 2018/19. The waiting time for stable new cases was long (median varied from 16 to 63 weeks), and was as long as 55 to 131 weeks in different clusters (Hospital Authority 2019b). Referral of all student cases to psychiatric SOPCs will further increase the burden of the public sector and this is against the direction of enhancing 
primary health care by the HKSAR Government. Prolonged waiting time may delay diagnosis confirmation and commencement of treatment, which may compromise the rehabilitation progress. Apart from the long waiting time, the out of campus location of the SOPCs and only fixed consultation appointments can be offered contribute to the low accessibility. Moreover, the student is required to submit the referral letter issued from family physicians to the relevant psychiatric SOPC in person for mental assessment by a nurse first, before the date of the first psychiatric appointment can be offered. This procedure requires strong motivation from the student. As a typical psychiatric SOPC is very busy with different types of psychiatric patients including both common mental disorders and psychotic disorders present in the waiting hall, students may have low acceptance due to disease stigma and unwilling to leave a formal record of psychiatric illness in the hospital system. In fact, because of these barriers, it would be very difficult, if not impossible to mobilize students to attend the psychiatric SOPCs. Furthermore, as the hospital psychiatrists do not belong to the university community, direct interdisciplinary communication between hospital psychiatrists and student counsellors, teachers from the university is less feasible and convenient.

\section{Proposal B: All Refer out to Private Psychiatrists}

This proposal will be welcome by private psychiatrists and the waiting time for the first appointment is short in general because of the availability of more psychiatrists in the private market. The students will have higher acceptance of seeking help from private psychiatrists when compared with public psychiatrists, as no official mental history will be made in the public mental health record. However, this proposal still shares similar drawbacks with proposal A, including the mental illness stigma concern, inconvenient interdisciplinary communication, out of campus location and fixed consultation appointments leading to lower accessibility of mental service to students. In particular, the major limitation of this proposal is the student financial concern on the relatively high consultation fees. According to a survey of 28 private psychiatrists in Hong Kong (Hong Kong Medical Association 2018), the median consultation fees for a psychiatric consultation per half hour is HKD 900 (roughly equivalent to USD 110). As the consultation time for a new case is at least one hour in general in private practice, a reasonable estimation for the total cost for a new case including both consultation fees and variable drug fees is higher than HKD 2000 (roughly equivalent to USD 260). As nearly 1.4 million of the city's residents were living below the official poverty line (HKSAR 2017), it is unrealistic to expect most of the students can afford the high consultation fees.

\section{Proposal C: Recruit Private Psychiatrist Stationed in Campus}

If a private psychiatrist is recruited and stationed in the University Health Service, it will be convenient for the students to attend and interdisciplinary communication will be more feasible. The students do not have a financial concern if the expenses of recruiting psychiatrist are to be supported by university central funding. As the caseload is not high enough to support a full-time psychiatrist, this proposal refers to recruiting the psychiatrist on a sessional basis per week at fixed consultation hours. However, in reality, students with emotional breakdown or active suicidal idea indicated for urgent 
psychiatric intervention can come to clinic anytime without following the fixed consultation timetable. If the students with an urgent psychiatric need cannot receive the timely psychiatric intervention because of the rigid consultation schedule, this will defeat the aim of this proposal. Furthermore, the expenses of psychiatrist recruitment increase with the number of sessions. Whether university central funding is available is another question. Furthermore, at present, all consultation rooms in University Health Service belong to medical officers who provide comprehensive medical service in general. If now the psychiatrist sits in one of the consultation rooms, it is predicted that people in the university community will soon notice the presence of this special consultation room. All patients entering this room may have the risk of being labelled having mental disorders. This is especially an important concern as the Chinese community generally have low acceptance of mental illnesses and it is highly likely that when waiting for psychiatrist consultation and drug collection in the waiting hall of University Health Service, patients will meet their classmates or colleagues whom they are working with daily in an enclosed university community. Causing potential embarrassment or even discrimination is predicted as patients' important concern for this proposal.

\section{Proposal D: Set up Mental Wellness Clinic under the Umbrella of in-Campus University Health Service}

If primary care professionals in the campus can manage students with common mental health problems, the strategy aligns with the plan of Hong Kong Government in enhancing primary healthcare and relieve the heavy burden of tertiary healthcare. The service will have higher accessibility because of the convenient location within campus. Case discussion among medical officer, student counsellor, program leader, and academic advisor, etc. is convenient as all professionals from different disciplines are university colleagues familiar with the university rules and settings. With prior student consent, when the medical opinion is required by various panels such as Board of Examiners when considering student's request for delayed examination, Student Hall Management when deciding on student's need for special hall residence, and Student Disciplinary Committee when handling student's disciplinary issues, direct interdisciplinary communication is feasible.

As the major service providers are full-time medical officers in the University Health Service, they share a sense of belonging to the university community and will be able to facilitate long-term health strategic planning. As these medical officers are trained as family physicians, the expensive medical manpower will not be idled when there is no mental patient because family physicians provide comprehensive medical care including both physical and mental problems resulting in cost-effectiveness. Moreover, there is no clear cut-off line between physical and psychosocial problems of students in many cases. For example, a student presented with severe acne (physical problem) may lead to depression (mental problem) as being teased and bullied by the peer (social problem), result in self-puzzling of whether should continue with the university education (spiritual problem). Family physicians who provide whole person care can manage these multi-aspects all together, hence avoiding over-fragmentation of problems in one student through unnecessary referrals. There are no financial concerns from both students and university management's perspectives, because no significant added 
monetary input is required for this regular medical service when compared with the significant expenses for proposal B (from students) and C (from university management). Arrangement for consultation appointments can be flexible as medical officers are full-time stationed in University Health Service instead of sessional basis for psychiatrist described in proposal $\mathrm{C}$.

In addition, the most important advantage of this proposal is the resolution of the stigma concern due to the "functional" nature of the service. University Health Service is a primary healthcare centre instead of a "mental health clinic". As there is no particular consultation room and no particular time-slots isolating students coming for mental health issues, the "strong label of mental disorders" is removed by "normalizing" patients coming for mental issues with all other patients coming for physical issues. Even meeting friends and colleagues in the common waiting area of the clinic, patients are no longer prone to embarrassment as there are no more "clues" suggesting of mental illnesses by observing which consultation room they walk into. However, the overall workload and work stress of the existing medical officers will increase. High quality experienced medical officers who are willing to and are capable of managing common mental disorders are required for this proposal.

\section{Introduction of the Mental Wellness Clinic within University Health Service}

After considering the various pros and cons of the aforementioned four proposals, proposal D has been carried out (Lo et al. 2018a). In one local university in Hong Kong, a Mental Wellness Clinic was established under the University Health Service in Hong Kong in September 2016. This is a ground-breaking multi-disciplinary service helping university students with mental health issues, including comprehensive mental health as well as clinical assessment, counselling, drug treatment, referral arrangement and follow up. It operates by a multidisciplinary coordinated healthcare model where medical officers (family physicians) of the University Health Service are supported by the nursing team to provide the service. Medical officers work closely with other disciplines such as student counsellors, university teachers, and external psychiatrists whenever necessary.

\section{The Pilot Experience of the Mental Wellness Clinic}

\section{Performance of the First Two Years}

The numbers of new case, headcount, and attendance for students attending the Mental Wellness Clinic in 2017 (the first year) and 2018 (the second year) are shown in Table 1. The fact of nearly 400 student headcount in 2018 and a $50 \%$ increase of student attendance from 2017 to 2018 reflects high student demand and challenging clinic workload. This also suggests acceptance of utilizing clinic service among the student population, which was previously one of the biggest concern for the stigma issue. Around half of the new cases are referred from student counsellors. The other half are shared by walk-in cases and intra- 
Table 1 Number of new case, headcount and attendance in 2017 and 2018

\begin{tabular}{lll}
\hline & 2017 & 2018 \\
\hline New Case & 258 & 297 \\
Headcount & 308 & 378 \\
Attendance & 1039 & 1544 \\
\hline
\end{tabular}

clinic referral, which means when a student attended University Health Service for physical issues initially but was detected by medical officer to have mental health concerns after opportunistic screening (i.e., early identification of at-risk cases). As one of the characteristics of student population, there were two peak utilization periods in October/November and March/April as shown in Table 2 corresponding to examination periods of the first and second semesters of the academic calendar respectively. Table 3 shows the most common diagnoses for students attended Mental Wellness Clinic, with anxiety, depression and subthreshold psychosomatic symptoms to be the most commonly seen problems. Regarding issues of maladjustment, the most commonly encountered problems included academic and work stress, family stress, and stress from peers and romantic relationship (Lo et al. 2018a). Around 15\% of cases belong to more complicated mental health illnesses who were referred to external psychiatrists for further management. This suggests that $85 \%$ of cases could be continuously managed by Mental Wellness Clinic or case closed due to recovery. From January to December 2018, there were 13 students presented with active suicidal ideation or recent suicidal attempt seek for urgent medical appointment in the Mental Wellness Clinic. Tragedies of potentially losing 13 student lives were prevented by timely medical intervention.

Table 2 Number of monthly new case, headcount and attendance in 2017 and 2018

\begin{tabular}{|c|c|c|c|c|c|c|}
\hline \multirow[b]{2}{*}{ Month } & \multicolumn{2}{|c|}{ New Case } & \multicolumn{2}{|c|}{ Headcount } & \multicolumn{2}{|c|}{ Attendance } \\
\hline & 2017 & 2018 & 2017 & 2018 & 2017 & 2018 \\
\hline Jan & 14 & 22 & 47 & 74 & 59 & 110 \\
\hline Feb & 23 & 15 & 50 & 68 & 69 & 89 \\
\hline Mar & 33 & 19 & 70 & 81 & 105 & 122 \\
\hline Apr & 22 & 31 & 59 & 96 & 90 & 129 \\
\hline May & 15 & 20 & 57 & 88 & 78 & 108 \\
\hline Jun & 14 & 24 & 56 & 87 & 79 & 127 \\
\hline Jul & 14 & 15 & 46 & 70 & 67 & 98 \\
\hline Aug & 16 & 18 & 48 & 79 & 66 & 123 \\
\hline Sep & 33 & 25 & 74 & 90 & 107 & 121 \\
\hline Oct & 29 & 43 & 77 & 115 & 109 & 177 \\
\hline Nov & 29 & 47 & 74 & 127 & 112 & 207 \\
\hline Dec & 16 & 18 & 70 & 100 & 98 & 133 \\
\hline
\end{tabular}


Table 3 Common diagnoses for students attended Mental Wellness Clinic

\begin{tabular}{ll}
\hline Diagnosis & Percentage \\
\hline Anxiety & 27 \\
Depression & 22 \\
Subthreshold psychosomatic symptoms & 20 \\
Sleep disturbances & 16 \\
Bipolar affective disorder & 5 \\
Eating disorder & 4 \\
Psychosis & 3 \\
Attention deficit hyperactivity disorder & 1 \\
Grief & 1 \\
Drug abuse & 1 \\
\hline
\end{tabular}

\section{Challenges Encountered}

\section{Increasing Clinic Workload with More "Prolonged Consultations"}

With the establishment of the new Mental Wellness Clinic, increasing workload for similar staffing of University Health Service is certainly the top challenge. As there were no external medical resources added regarding this new mental health service, internal manpower redistribution was performed within University Health Service. If the average consultation time for a student with simple ailments such as common cold symptoms is $5-10 \mathrm{~min}$, the average consultation time for a student newly came for emotional disturbances is at least $30 \mathrm{~min}$. Prolonged consultation time is required for allowing patients' ventilation of emotion, rapport building, proper mental health assessment, case conceptualization, education of drug use before prescription of psychiatric medication, brief psychological intervention, and writing up of medical report when necessary. In order to spare, some precious doctors' consultation timeslots to satisfy the need of this prolonged consultation demand from an already fully booked busy University Health Service, the nursing team has taken up some simpler and low risk vaccination-counselling tasks after training. For example, pre-HPV vaccination counselling for all students was performed by medical officers with each student required a 10-min individual medical consultation timeslot in the past. This has been replaced by conducting HPV vaccination campaigns with health talks and individual counselling when necessary conducted by advanced practice nurse under the supervision from a medical officer. For around 700 students received HPV vaccination in 2018, an estimation of 1400 medical consultation timeslot with 5 min each was spared to accommodate the provision of more complicated cases such as mental health consultations. As 50\% of new mental cases attended Mental Wellness Clinic directly without referrals from student counsellors, advanced practice nurses are responsible for conducting an immediate mental health assessment upon student arrival, to triage the new cases into urgent, semi-urgent and routine cases. According to service pledge of Mental Wellness Clinic, new cases should be offered a medical appointment within the same day, 2 days and 1 week respectively, and so far, this service pledge can be achieved completely by an effective triage system and flexible logistics. 


\section{Increasing Mental Case Complexity}

Complicated mental cases include diagnostic uncertainty, recurrent suicidal thoughts, depression with suboptimal response to first line medical treatment, suspected bipolar affective disorder, severe eating disorders, and psychotic disorders, etc. Timely psychiatric referral is indicated because it is beyond the capacity of primary care management. As mentioned in proposal A and B, the major barriers for public psychiatry referral and private psychiatry referral include long waiting time and financial concern respectively. In order to strengthen the referral logistics to public psychiatrists, representatives from Mental Wellness Clinic visited the Chief of Service of Psychiatry (of the relevant cluster with the university) under Hospital Authority for further discussion. With the support from The Hong Kong College of Psychiatrists, coordinator of Mental Wellness Clinic had communication with more than 30 private psychiatrists for discussion on the referral logistics and financial concerns of the students. Most of the medical officers of Mental Wellness Clinic have higher professional qualifications such as Specialists in Family Medicine, with mental health training during previous rotation to psychiatric hospitals. Psychiatrists are regularly invited to the University Health Service to conduct update seminar for Continuous Medical Education CME. Drug varieties of the in-house dispensary have been expanded after obtained expert opinion from psychiatrists.

\section{Higher Stress for Medical Officers}

At the beginning stage of Mental Wellness Clinic, there were only three medical officers assigned to manage mental cases. As both the caseload and case complexity increase as evidenced by the nearly 50\% increase of student attendance from 2017 to 2018 , mental cases are shared by seven medical officers by now. This can avoid potential burnout of any single medical officer because over-working with mental cases may increase the risk of psychological exhaustion of the health professional. For high-risk cases without immediate support from external psychiatrist available, medical officers are encouraged to have close communication with the respective student counsellors so that both professionals can provide mutual support to each other in times of stress. Medical case conferences for selected difficult cases have been organized among all medical officers of the Mental Wellness Clinic with support from external psychiatrists. A default call-back system has been set up by an advanced practice nurse and her nursing team who is responsible to call back all default cases on a monthly basis after confirmation with the respective case medical officer. This systematic measure can minimize the risk of missing patients who default follow up appointment due to mood deterioration, and decrease the stress of case medical officers who may be unable to remember the follow up appointments of all patients especially during peak seasons. The importance of work-life balance and the concept of medical humanities are re-visited from time to time, which may decrease the chance of burnout among medical professionals (Lo et al. 2018b).

\section{Myths and Stigma about Mental Illnesses in the Community}

Instead of waiting for patients coming into the consultation room by themselves, staff from Mental Wellness Clinic reach out to the university community for promotion of mental wellness service in collaboration with other disciplines. Medical and nursing 
professionals are invited to participate in orientation talks to meet over 4400 new students at the beginning of the semester. Health education videos on mental wellness are produced by University Health Service and student ambassadors, which are spread among the students with the use of social media platforms such as Facebook and YouTube. Representatives from Mental Wellness Clinic also visited all the six faculties and two schools of the university, introducing mental health service to the respective Deans and Heads seeking for their support. Furthermore, a medical professional from the Mental Wellness Clinic participates in the training workshops of the academic advisors, increasing their awareness of mental health problems when dealing with

students. Regarding individual counselling in the medical consultation room, it is emphasized that having mood symptoms is not the fault of the patient; they are usually reversible and will improve with treatment. For the more serious cases, parents are invited to participate in the overall management, such as supervision of drug compliance, if prior consent from the student is obtained.

\section{Future Direction}

In order to achieve higher coverage of mental wellness promotion to all University students (i.e., primary prevention), medical representatives of University Health Service will have active participation in the teaching of Healthy Lifestyle Program, which is a compulsory subject under the General University Requirements for all undergraduate students. Medical participation will be in the form of a) writing health articles with local context tailor made to university students in Hong Kong as compulsory e-learning material followed by multiple-choice assessments to facilitate in-depth understanding b) conducting the mental health session by a medical professional in every introductory lecture throughout the academic year. This hopefully can increase the coverage to nearly $100 \%$ of all undergraduate students via the platform of this compulsory program. Subjective outcome evaluation showed that the students enjoyed the e-learning materials and the lectures. They also acquired more knowledge on mental wellness and some of the more prevalent mental health problems amongst university students.

In terms of secondary prevention (i.e., early identification of the at-risk cases), early screening using DASS (Depression, Anxiety, Stress Scale), mental health-screening questionnaire for all new students is currently conducted by the Office of Counselling and Wellness. University Health Service will continue to support by providing mental health assessment to the screened high-risk cases upon referral by student counsellors. Special attention should be paid to potential high-risk groups such as non-local students and students under academic probation, etc. Further work with the teachers and academic counsellors is needed for such cases.

Obviously, while the proposed model is sound on the conceptual level, more evaluation is needed to understand the impact of the intervention. There are at least three areas we can consider. First, subjective outcome evaluation using rapid assessment instruments can be used to understand the view of the clients on the service provided and the perceived benefits of the intervention. This method is commonly used by different professionals in the medical setting. Second, qualitative evaluation such as individual interviews can be conducted to understand the experiences of the different stakeholders. In particular, we should pay attention to the work-life balance and burnout 
status of colleagues engaging in this program. Third, with proper design, collection of pretest and posttest data using mental health status and well-being measures may give some evidence on the effectiveness of the intervention. Of course, if resources permit, we should collect longitudinal data on the impact of this service on the students in the long run. In short, the proposed strategy is an exciting possibility for helping students with mental health challenges. Although the experience is based in Hong Kong, it has high relevance to mental health service for college students in other parts of the world.

\section{Compliance with Ethical Standards}

Conflict of Interest The authors declare that they have no conflict of interest.

Open Access This article is distributed under the terms of the Creative Commons Attribution 4.0 International License (http://creativecommons.org/licenses/by/4.0/), which permits unrestricted use, distribution, and reproduction in any medium, provided you give appropriate credit to the original author(s) and the source, provide a link to the Creative Commons license, and indicate if changes were made.

\section{References}

Auerbach, R., Alonso, J., Axinn, W., Cuijpers, P., Ebert, D., Green, J., et al. (2016). Mental disorders among college students in the WHO world mental health surveys. Psychological Medicine, 46(14), 2955-2970.

Breslau, J., Lane, M., Sampson, N., \& Kessler, R. (2008). Mental disorders and subsequent educational attainment in a US national sample. Journal of Psychiatric Research, 42(9), 708-716.

Department of Health, HKSAR. (2019). Student Health Service. Retrieved from Department of Health: https://www.dh.gov.hk/english/main/main_shs/main_shs.html. Accessed 05 June 2019.

HKSAR. (2017). Hong Kong poverty situation report. Hong Kong.

HKSAR. (2019). Overview of the healthcare system in Hong Kong. Retrieved from Gov HK: https://www. gov.hk/en/residents/health/hosp/overview.htm. Accessed 05 June 2019.

Ho, E., Au, S., Bedford, C., and Cooper, J. (2003). Mental health issues for Asians in New Zealand: A literature review. Mental Health Commission. Migration Research Group.

Hong Kong Medical Association. (2018). Hong Kong medical association Doctors' fees survey 2018. Hong Kong. Retrieved from http://www.hkma.org/english/newsroom/news/20190125\%20HKMA\%20 Doctors\%20Fees\%20Survey\%202018.pdf. Accessed 25 June 2019.

Hospital Authority. (2011). Hospital Authority mental health service plan for adults 2010-2015. Hong Kong.

Hospital Authority. (2019a). HA Convention Masterclass 2. Retrieved from integrated mental health Programme for common mental disorder patients in GOPCs: http://www3.ha.org. hk/haconvention/hac2014/proceedings/downloads/MC2.3.pdf. Accessed 28 June 2019.

Hospital Authority. (2019b). Specialist Outpatient Clinics. Retrieved from waiting time for new case booking at psychiatric specialist outpatient clinics: http://www.ha.org.hk/visitor/sopc_ waiting_time.asp?id=7\&lang=ENG. Accessed 27 June 2019.

Hospital Authority, HKSAR. (2019). Psychiatric SOPC. Retrieved from List of SOPCs: http://www.ha.org. hk/visitor/ha_visitor_index.asp?Content_ID=200252\&Lang=ENG\&Dimension=100\&Parent_ID=10053. Accessed 05 June 2019.

Ibrahim, A., Kelly, S., Adams, C., \& Glazebrook, C. (2012). A systematic review of studies of depression prevalence in university students. Journal of Psychiatric Research, 47(3), 391-400.

Kessler, R., Walters, E., \& Forthofer, M. (1998). The social consequences of psychiatric disorders, III: Probability of marital stability. The American Journal of Psychiatry, 155(8), 1092-1096.

Kessler, R., Berglund, P., Demler, O., Jin, R., Merikangas, K., \& Walters, E. (2005). Lifetime prevalence and age-of-onset distributions of DSM-IV disorders in the National Comorbidity Survey Replication. Archives of General Psychiatry, 62(6), 593-602. 
Lam, L., Wong, C., \& Wang, M. (2015). Prevalence, psychosocial correlates and service utilization of derpessive and anxiety disorders in Hong Kong: The Hong Kong mental MOrbidity survey. Social Psychiatry and Psychiatric Epidemiology, 50(9), 1379-1388.

Lo, D., Wong, H., Lam, J., \& Shek, D. (2018a). Common mental health challenges in a university context in Hong Kong: A study based on a review of medical record. Applied Research in Quality of Life, 1-12.

Lo, D., Wu, F., Chan, M., Chu, R., \& Li, D. (2018b). A systematic review of burnout among doctors in China: A cultural perspective. Asia Pacific Family Medicine, 2019, 17-13.

Lun, K., Chan, C., Ip, P., Ma, S., Tsai, W., Wong, C., et al. (2018). Depression and anxiety among university students in Hong Kong. Hong Kong Medical Journal, 24(5), 466-472.

Monash University. (2019). Health and wellbeing. Retrieved from Medical team: https://www.monash.edu/ health/medical-services/team.

National University of Singapore. (2019). University health Centre. Retrieved from Specialist Clinic: http:// www.nus.edu.sg/uhc/services/general-health/specialist-clinic.

Osby, U., Brandt, L., Correia, N., Ekbom, A., \& Sparen, P. (2001). Excess mortality in bipolar and unipolar disorder in Sweden. Archives of General Psychiatry, 58(9), 844-850.

Penn State University. (2018). Center for Collegiate Mental Health Annual Report 2018.

Shek, D. T. L. (1990). Mental health knowledge of Chinese secondary school students in Hong Kong. CUHK Education Journal, 18(2), 143-152.

Shek, D. T. L., \& Liang, L. Y. (2018). Psychosocial factors influencing individual well-being in Chinese adolescents in Hong Kong: A six-year longitudinal study. Applied Research in Quality of Life, 13(3), 561-584. https://doi.org/10.1007/s11482-017-9545-4.

Shek, D. T. L., \& Siu, A. M. H. (2019a). "UNHAPPY” environment for adolescent development in Hong Kong [special issue]. Journal of Adolescent Health, 64(6), S1-S4.

Shek, D. T. L., \& Siu, A. M. H. (2019b). Adolescent mental health policy and Services in Hong Kong: Seven unresolved problems waiting for solutions. Journal of Adolescent Health, 64(6), S5-S9.

Shek, D. T. L., Yu, L., Wu, F. K. Y., Zhu, X., \& Chan, K. H. Y. (2017). A 4-year longitudinal study of wellbeing of Chinese university students in Hong Kong. Applied Research in Quality of Life, 12(4), 867-884.

The University of Tokyo. (2019). Division for counseling and support. Retrieved from Office for Mental Health Support: http://dcs.adm.u-tokyo.ac.jp/mhs/en/. Accessed 28 June 2019.

Thompson, A., Hunt, C., \& Issakidis, C. (2004). Why wait? Reasons for delay and prompts to seek help for mental health problems in an Australian clinical sample. Social Psychiatry and Psychiatric Epidemiology, 39(10), 810-817.

Turner, J., Leno, E., \& Keller, A. (2013). Causes of mortality among American college students: A pilot study. Journal of College Student Psychotheraphy, 27(1), 31-42.

University of Bristol. (2019). Students' Health Services. Retrieved from Mental Health Service: http://www. bristol.ac.uk/students-health/services/mental-health/. Accessed 28 June 2019.

University of Michigan. (2019). University Health Service. Retrieved from Mental Health Service at UHS: https://www.uhs.umich.edu/mentalhealthsves. Accessed 28 June 2019.

Wong, J., Cheung, E., Chan, K., Ma, K., \& Tang, S. (2006). Web-based survey of depression, anxiety and stress in first-year tertiary education students in Hong Kong. Australian and New Zealand Journal of Psychiatry, 40(9), 777-782.

Wong, P., Arat, G., Rehnu, M., Xie, K., \& Borschel, M. (2019). Evaluation of a mental health course for stigma reduction: A pilot study. Cogent Psychology, 6(1), 1595877.

World Health Organization. (2013). Investigating in mental health: Evidence for action.

World Health Organization. (2017). Depression and other common mental disorders: Global Health estimates. Geneva: World Health Organization.

Publisher's Note Springer Nature remains neutral with regard to jurisdictional claims in published maps and institutional affiliations. 